\title{
Ordre et chaos
}

La réforme du système espagnol

Continuity and disarray. Reforming the Spanish education system

Orden y desorden. La reforma del sistema español

\section{Amalia Ayala de la Peña et Ángel González Hernández}

Traducteur : Yolanda Christon

\section{(c) OpenEdition}

\section{Journals}

Édition électronique

URL : https://journals.openedition.org/ries/2532

DOI : 10.4000/ries.2532

ISSN : 2261-4265

Éditeur

France Education international

Édition imprimée

Date de publication : 1 juin 2000

Pagination : 83-95

ISSN : $1254-4590$

Référence électronique

Amalia Ayala de la Peña et Ángel González Hernández, "Ordre et chaos », Revue internationale d'éducation de Sèvres [En ligne], 26 | juin 2000, mis en ligne le 01 juin 2003, consulté le 08 décembre 2022. URL : http://journals.openedition.org/ries/2532 ; DOI : https://doi.org/10.4000/ries.2532 


\section{Ordre et chaos ${ }^{1}$}

\section{La réforme du système espagnol}

Amalia Ayala de la Peña

Ángel González Hernández

\section{Résumé}

Dans le contexte des vastes réformes qu'a connues l'Espagne depuis le début des années soixante-dix, la mise en place d'un Institut national de la qualité et de l'évaluation ne s'est pas effectuée sans heurts ni problèmes. L'accueil réservé au récent rapport publié par cet organisme sur l'enseignement secondaire obligatoire en est la preuve, illustrée ici par les témoignages de la presse.

Continuity and disarray. Reforming the Spanish education system

The creation of a National Quality and Evaluation Institute ushered in by the vast programme of reform introduced in Spain in the early 1970 s did not go smoothly. This was illustrated by the reception given to one of the Institute's recent reports on Spain's public secondary schools, which was the subject of fierce controversy in the Spanish press.

Orden y desorden. La reforma del sistema español

En el marco de amplias reformas que fueron adoptadas en España desde el principio de los años 1970, el establecimiento de un Instituto Nacional de Calidad y Evaluación no se llevó a cabo sin dificultades. Testimonios de la prensa ponen de realce las reacciones ante el reciente informe sobre enseñanza segundaria, publicado por este organismo. 
"Elohîm forme de la glèbe tout animal du champ, tout volatile des ciels, il les fait venir vers le glébeux pour voir ce qu'il leur criera. Tout ce que le glébeux crie à l'être vivant, c'est son nom. "

La Genèse 2-19, traduction d'André Chouraqui, 1992

\section{Il était une fois un pays de réformes éducatives}

Depuis le début des années soixante-dix, avec la promulgation de la loi générale d'éducation (LGE) ${ }^{2}$ de 1970, faisant suite à la publication du livre blanc de l'éducation de 1969, l'Espagne aborde le XXI ${ }^{\mathrm{e}}$ siècle dans un processus continu de réformes à tous les niveaux de l'édifice scolaire. La démocratisation politique, qui a débuté au milieu des années soixante-dix, a été le terrain favorable à l'orientation et à l'organisation de l'appareil scolaire, dans un pays où le développement économique et démographique imposait de grandes réformes structurelles de l'éducation.

Au cours des deux décennies suivantes, la loi générale de 1970 et sa structure organique a servi de cadre statutaire (excepté certains décrets favorisant la démocratisation de sa structure). Ce n'est qu'avec l'approbation de la loi d'ordonnancement général du système éducatif (LOGSE) ${ }^{3}$

Ce n'est ni le moment de faire des analyses socio-économiques, ni de commenter les résultats d'une évaluation $d u$ système éducatif secondaire aux facteurs multiples ; à chacun son interprétation de 1990 que la nouvelle structure scolaire a commencé à s'implanter progressivement. Entre-temps, véhiculées par les avatars politiques de la prétendue «transition " démocratique, d'autres lois ont tenté de pallier et de révéler les déficiences de la LGE comme, par exemple, la loi organique du 19 juin 1980 qui réglementait le statut des établissements scolaires (LOECE) ${ }^{4}$. Ordonnancement "démocratique», mais également fortement idéologique, elle s'efforçait de masquer les connotations non démocratiques de la LGE, encore sous le régime de Franco, et parallèlement de consolider certains bénéfices des établissements privés. L'intégralité du titre III sur "Les établissements privés » est consacrée aux pri-vilèges acquis par les collèges dirigés par des religieux. Ces établissements font partie d'un réseau privé d'enseignement que les religieux dominent avec une majorité absolue, surtout dans l'enseignement secondaire qui s'appelle encore "enseignements moyens». Les dispositions finales annulent tous les

2 Ley General de Educación.

3 Ley de Ordenación General del Sistema Educativo.

4 Ley de los Estatutos de Centros Escolares. 


\section{dossier}

articles obsolètes de la loi précédente et d'autres lois encore en vigueur "dans la mesure où elles sont contraires à la loi actuelle». Cette loi de courte durée a bouleversé le monde politique. Elle a réussi à rompre le consensus des partis politiques pour la transition démocratique, alors que d'autres lois de cette époque, pourtant plus polémiques, comme la loi sur le divorce et ou sur la réforme fiscale, n'y étaient pas parvenues.

La réplique politique à la LOECE émane d'une loi idéologique sur l'éducation, approuvée par une large majorité après la victoire socialiste de 1982. Elle a inauguré un changement qui s'est concrétisé par la loi organique du droit à l'éducation $(\mathrm{LODE})^{5}$ de 1985 , régulatrice du droit à l'éducation. Cette nouvelle loi - nécessaire et polémique, pacificatrice d'une guerre scolaire qui n'avait pas éclaté en Espagne à la fin du XIX ${ }^{e}$ siècle - confortait l'ancienne LGE dans ses effets structurels et organisationnels. Le nouveau cabinet, qui devait s'occuper en urgence de la réforme de l'université, a rédigé dans un temps record la loi organique de réforme universitaire $(\mathrm{LRU})^{6}$, votée le 25 aout 1983, huit mois après l'investiture du nouveau gouvernement. Le problème universitaire avait été sous-jacent au cours de la dernière décennie du franquisme ${ }^{7}$. Trois projets de loi d'autonomie universitaire avaient été rejetés pendant la transition et leurs auteurs, les trois ministres des gouvernements du centre d'Adolfo Suárez, destitués.

Lentement mais sûrement, la troisième majorité socialiste a rédigé la loi organique (LOGSE) du 3 octobre 1990, qui prône un changement d'ordre structurel du système éducatif obligatoire. En cours d'application et à peine totalement implantée, cette loi a fait l'objet d'une demande d'évaluation de la part de Esperanza Aguirre, ministre du premier gouvernement conservateur de José Maria Aznar : simple vérification routinière de qualité ou intention occulte de démanteler la structure scolaire établie par les socialistes afin de la substituer par une autre qui épouserait mieux son idearium politique?

\section{Audi vocem meam : les sirènes de la qualité éducative en Espagne}

Les différents moyens d'information déterminent une variété de modèles d'interprétation du type d'évaluation mais visent toutes la prise de décisions. Le modèle d'évaluation systématique de l'éducation en Espagne émane de la LOGSE et se concrétise par la création, en 1993, de l'Institut national de la qualité et de l'évaluation (INCE) ${ }^{8}$. L'article 62.3 stipule clairement que l'« éva-

\footnotetext{
5 Ley Orgánica del Derecho a la Educación.

6 Ley de Reforma Universitaria.

7 González Hernández A., "La cuestión universitaria: asignaturas pendientes ", Revista Española de Educación Comparada, 1999, n 5, pp. 37-57.

8 Instituto Nacional de Calidad y Evaluación.
} 
luation générale du système éducatif sera réalisée par l'INCE. Le gouvernement, après avoir consulté les communautés autonomes, déterminera l'organisation de l'institut et lui accordera tous les moyens nécessaires à son bon fonctionnement.» Son objectif et sa marge de manœuvre sont clairement définis dans le premier point de l'article 62 de la LOGSE : «L'évaluation du système éducatif visera à l'adéquation constante de ce système aux demandes sociales et aux besoins éducatifs et portera sur les élèves, le corps enseignant, les établissements, les procédures éducatives et l'administration même. »

\section{Au commencement de la qualité était la pédagogie}

Avant l'INCE - dont le décret royal 928/1993 entérine la création et spécifie les fonctions, la composition, les organes de gestion et l'organisation - il y avait le Centre national de documentation éducative (CIDE) ${ }^{9}$, créé en 1983. Bien que l'évaluation ne soit pas son objectif principal, il a contribué à travers ses programmes à l'évaluation du système éducatif espagnol dans certaines études comme le Rapport de base : Études pour l'évaluation du système éducatif espagnol ${ }^{10}$ qui est devenu un guide de référence sur le système éducatif avant la LOGSE.

Cependant, avant la LGE, la recherche éducative « en tant qu'information sur le système d'enseignement " s'effectuait dans les départements de pédagogie qui ne constituaient alors qu'une branche du gigantesque arbre des facultés de philosophie et de lettres. Nous avons souligné que la parution en Espagne du livre blanc sur l'éducation (1969) a révélé la nécessité d'entreprendre une réforme totale du système éducatif ; il semblait approprié que le ministère et les universités élaborassent ensemble une politique éducative nationale. C'est à ce titre que sera créé le Centre national de recherches pour le développement de l'éducation (CENIDE) ${ }^{11}$ comme noyau d'un réseau régional d'Instituts de sciences de l'éducation (ICEs) ${ }^{12}$ liés aux universités ${ }^{13}$. Quelques années après la création du CENIDE, destiné à épauler la réforme de la LGE, cet organisme a été remplacé par l'Institut national des sciences de l'éducation (INCIE) ${ }^{14}$. Bien que rattaché au ministère de l'Éducation et de la Science, il possède cependant sa propre identité juridique et un capital financier indépendant du ministère, et sera donc à même

9 Centro Nacional de Documentación Educativa.

10 OCDE, 1991.

11 Centro Nacional de Investigaciones para el Desarrollo de la Educación.

12 Institutos de Ciencias de la Educación.

13 Ayala A. y González A., "Formation des enseignants ou déformation professionnelle ? Le jeu des réformes éducatives en Espagne ", Revue internationale d'éducation, 1999, nº 20, pp. 37-59.

14 Instituto Nacional de Ciencias de la Educación. 
d'innover et de former les enseignants. Son articulation ayant été trop rapidement définie, une contradiction existait entre le fonctionnement théorique de cet institut et la réalité vécue dans les établissements. La situation s'est aggravée avec la séparation administrative qu'entraîna la création d'un ministère des Universités, distinct du reste du système éducatif. Lors de sa fermeture en 1980, les fonctions de recherches ont été transférées au ministère des Universités et de la Recherche, et les autres directement au ministère de l'Éducation.

Comme suite à tous les changements départementaux au sein des ministères, le CIDE fut créé en 1983 afin de superviser la recherche éducative, la documentation, la bibliothèque et les archives du ministère de l'Éducation qui avait à nouveau la charge des universités. La situation est restée inchangée jusqu'à la création de l'INCE, rattaché au ministère de l'Éducation sous le premier gouvernement Aznar. La création d'un ministère de l'Innovation et de la Technologie chargé de co-diriger la recherche avec le ministère de l'Éducation remet en cause l'utilité de l'INCE. Chronique d'une mort annoncée?

\section{La fonction crée l'organe : I'INCE}

L'évaluation de l'éducation est inscrite dans la LOGSE puisque l'article 55 stipule que les pouvoirs publics doivent s'engager à améliorer l'enseignement. Nous avons déjà signalé que l'article 62 confie clairement l'évaluation du système éducatif espagnol à l'INCE. Le troisième point de cet article entérine la création de l'institut: «L'évaluation générale du système éducatif sera réalisée par l'INCE. Le gouvernement, après avoir consulté les communautés autonomes, déterminera l'organisation de l'Institut et lui accordera tous les moyens nécessaires à son bon fonctionnement. »

L'objectif de l'INCE est bien évidemment de réaliser une évaluation générale du système éducatif, sans contrecarrer les évaluations du système faites par les administrations éducatives dans leurs propres domaines de compétences. L'Institut se charge également :

- d'élaborer des systèmes d'évaluation des enseignements réglementés par la loi et les établissements qui les proposent ;

- de faire des recherches, des études et des évaluations du système éducatif et, en général, de proposer aux administrations éducatives toutes les initiatives et les suggestions qui peuvent contribuer à l'amélioration de la qualité et de l'enseignement (LOGSE, article 62.4).

Le 18 juin 1993, le décret royal 928/1993 a élargi et réglementé les fonctions de l'organe d'évaluation. Rattaché à l'administration générale de l'État, l'INCE est responsable de l'évaluation générale du système éducatif.

En vertu de l'article 3 de ce décret, les fonctions suivantes lui ont été attribuées :

- évaluer le degré d'acquisition des enseignements minima fixés à chaque niveau, étape, cycle et degré du système éducatif, dans le cadre du pro- 
gramme défini par les administrations éducatives et de sa mise en place dans les établissements scolaires ;

- évaluer les réformes générales du système éducatif, de même que la structure, la portée et les résultats des innovations d'ordre général introduites dans celui-ci ;

- élaborer un système national d'indicateurs qui permette d'évaluer le degré d'efficacité et d'efficience du système éducatif ;

- coordonner au niveau national la participation à des études internationales d'évaluation ;

- élaborer des systèmes d'évaluation pour les différents enseignements réglementés par la LOGSE et les établissements qui les proposent ;

- coopérer, si la participation de l'institut est requise et en fonction de ses disponibilités et de ses missions, avec les administrations, les institutions et les organismes qui assument une quelconque responsabilité en évaluation éducative ;

- informer les différents secteurs de la société sur le fonctionnement et les résultats du système éducatif, en fonction des critères adoptés par la Conférence sectorielle d'éducation ;

- donner et échanger des informations avec les administrations concernées afin de faciliter la prise de décisions ;

- publier et diffuser les résultats des évaluations et des innovations réalisées dans le domaine de l'évaluation, en fonction des critères adoptés par la Conférence sectorielle d'éducation, sans contrecarrer le travail et les publications des administrations dans leurs propres domaines de compétences.

La structure organique de l'INCE est chapeautée par un directeur, nommé par le ministre de l'Éducation et de la Culture, et par un comité scientifique, composé d'un président et de six membres au maximum, désignés par le ministre de l'Éducation et de la Culture à la suite d'une audience accordée lors de la Conférence sectorielle d'éducation. Les membres du comité doivent jouir d'un prestige scientifique et professionnel dans le domaine de l'évaluation et/ou de la recherche éducatives.

Le directeur de l'INCE est également secrétaire du "Conseil recteur " qui compte les membres suivants :

- le président, qui est le secrétaire d'État à l'éducation,

- le directeur général de la rénovation pédagogique, le directeur général de la coordination et de la haute inspection, le directeur général de la formation professionnelle réglementée et de la promotion éducative, un haut représentant du directeur général ou de son homologue, désigné par chaque administration éducative. Ce dernier représente les administrations des communautés autonomes qui disposent déjà de compétences en éducation.

Grâce à la présence des représentants technico-politiques des gouvernements autonomes, le Conseil recteur de l'INCE est légitime puisqu'il couvre l'ensemble du territoire national, sans contrecarrer la capacité évaluatrice des autorités autonomes des régions dans l'exercice de leurs compétences éducatives. 
Le directeur de l'INCE représente l'institut. Il assure son fonctionnement et assume la responsabilité du développement de son activité. L'article 7 du décret royal énumère ses fonctions :

- représenter l'institut,

- proposer au Conseil recteur les plans d'action annuels et pluriannuels de l'institut,

- développer les plans d'action approuvés par la Conférence sectorielle d'éducation,

- veiller à l'accomplissement des décisions du Conseil recteur,

- informer le Conseil scolaire sur l'état d'avancement des travaux effectués par l'institut conformément au paragraphe 7 de l'article 3 du décret qui fixe les fonctions de l'INCE,

- assurer un certain nombre de fonctions qui lui sont expressément confiées.

Sur son site $\mathrm{Web}^{15}$, l'INCE présente la planification des activités suivantes pour l'année 1999-2000:

Actions continues :

- élaboration d'un système national d'indicateurs de l'éducation,

- construction d'une banque d'items et de tests de rendement,

- conception et développement de programmes de formation d'experts en évaluation,

- publication de la Revue d'éducation.

Projets spécifiques :

- étude sur l'enseignement et l'apprentissage de la langue anglaise en Espagne,

- deuxième étude d'évaluation de l'éducation primaire,

- évaluation de l'éducation secondaire obligatoire (quatrième année),

- diagnostic de la fonction de direction dans les établissements à fonds publics.

Participation à des études internationales :

- projet international d'indicateurs des systèmes éducatifs (INES) de l'OCDE, projet international pour la production d'indicateurs de résultats éducatifs des élèves (PISA) de l'OCDE, étude d'établissements d'éducation secondaire post-obligatoire,

- programme d'évaluation de la qualité de l'éducation (OEI),

- participation à d'autres projets bilatéraux ou multilatéraux.

L'INCE est indéniablement, de par sa structure et ses fonctions, un organe technico-politique : il n'aurait sans doute pas pu en être autrement. Cette double fonction le condamne à être la cible des invectives des uns et des autres, il se situe à la croisée des tirs entre professionnels et politiciens "professionnalisés ». La polémique précède toute action. 


\section{Le diagnostic de l'école secondaire obligatoire}

La LOGSE met en place une nouvelle structure de l'éducation en Espagne : un concept d'enseignement par cycles fondé sur une école primaire jusqu'à 12 ans (6-12 ans), puis un enseignement secondaire au terme duquel l'élève accède aux enseignements professionnels ou à l'université. L'enseignement secondaire se décompose en plusieurs niveaux, les plus structurés étant ceux marqués par un premier cycle secondaire obligatoire (12-16 ans) et un second cycle non obligatoire, le Bachillerato (16-18 ans). L'enseignement secondaire obligatoire (ESO) est le principal changement organique instauré par la LOGSE (1990) par rapport à l'organisation de la LGE (1970). L'ancienne structure scolaire prolongeait l'enseignement élémentaire jusqu'à 14 ans, appelé enseignement général de base et dispensé par une école obligatoire. Sous la tutelle de la Direction générale des enseignements moyens, une sorte d'enseignement secondaire, appelé le Bachillerato Unificado Polivalente (BUP), s'est alors annexé à l'enseignement général de base. Il ne fut, cependant, ni unique ni même polyvalent car il ne conduisait comme propédeutique qu'à l'université.

En prolongeant le caractère obligatoire de l'éducation jusqu'à 16 ans, la LOGSE a restructuré le parcours scolaire. L'ancienne école élémentaire est devenue l'école primaire. Les deux dernières années de l'enseignement général de base (LGE, 1970) ont été transférées dans le premier cycle de l'enseignement secondaire à l'instar des deux premières années de l'ancien Bachillerato (14-16) afin de créer une école secondaire obligatoire.

Ce n'est que quelques années après l'application de la LOGSE que le chevauchement entre les deux organisations a entraîné, comme toujours, une kyrielle de comparaisons haineuses entre les «conservateurs » de l'ancien système et les "progressistes " du nouveau - conflits plus idéologiques et politiques que rationnels et techniques. D'une part, les élèves du même âge dans deux types d'écoles différents devraient avoir la même obligation légale face à leurs programmes respectifs; d'autre part, la situation est tendue entre les défenseurs du nouveau-né - ce système largement conçu par les socialistes et tardivement implanté par leur dernier gouvernement - et les détracteurs du "monstre", appartenant pour la plupart à la majorité conservatrice qui voudrait se débarrasser de ce système qui émane des socialistes auxquels ils attribuent déjà les lois scolaires (LODE, 1985 ; LOGSE, 1990) adoptées après l'urgente réforme universitaire (LRU, 1983).

Lorsque les conservateurs (Partido Popular) ont pris le pouvoir en 1996, un manque d'enthousiasme a rejailli sur la nouvelle administration en raison de l'application de la consensuelle réforme LOGSE. C'est également dans ce climat que s'effectua le changement de direction de la haute administration avec les inévitables nominations duelles entre capacité professionnelle nécessaire et pouvoir discrétionnaire de la «lutte » politique. Il était prévisible que l'INCE, récem- 
ment créé, changeât également d'orientation avec la nomination du professeur José Luís García Garrido, professionnel de l'éducation réputé, spécialiste international en éducation comparée, professeur à l'université nationale d'éducation à distance.

L'INCE s'est lancé dans un projet ambitieux mais nécessaire : évaluer, estimer ou "calibrer » l'étape chronologique des 14-16 ans du système éducatif espagnol qui correspond, d'une part, au deuxième cycle de l'enseignement secondaire obligatoire de la LOGSE et, d'autre part, aux $1^{\mathrm{e}}$ et $2^{\mathrm{e}}$ années de BUP et à la Formation professionnelle (FP) de l'ancien programme. Cette étude sur la qualité de l'éducation et du système éducatif pour les élèves espagnols de 14 à 16 ans a débuté en 1997, les résultats ont été divulgués le 17 juin 1998 en six volumes ${ }^{16}$. Le directeur de l'INCE, auteur de Macrodiagnóstico del Sistema educativo, a présenté sa démission après la publication de l'étude.

La presse connaissait depuis des mois le contenu du Rapport officiel présenté par $\mathrm{M}^{\mathrm{me}}$ la ministre le 17 juin 2000. Le milieu politique avait déjà pensé l'utiliser comme une arme. Avant même que les premiers résultats soient révélés, début mars, certaines données avaient été manipulées afin de conforter l'une ou l'autre des thèses possibles : conserver ou démanteler l' « édifice LOGSE ». $\mathrm{M}^{\mathrm{me}}$ la ministre, Esperanza Aguirre, a déclaré le 17 juin : «Nous ne pouvons ni accepter ni justifier la faiblesse des résultats de l'éducation. » Le gouvernement a diffusé les informations suivantes :

- $25 \%$ des élèves de 14 ans et $33 \%$ des élèves de 16 ans n'atteignent pas les niveaux minimums de connaissances;

- les « humanités " et les mathématiques doivent être consolidées, selon la ministre.

Esperanza Aguirre semble s'appuyer sur ce Rapport pour donner une nouvelle impulsion politique à son projet de réforme des humanités à l'école, qui avait fait l'objet d'un rejet unanime - plus politique que rationnel - de la part de l'opposition, auparavant au pouvoir. La réforme des humanités mérite d'autres fleurs que celles déposées sur les tombes des ministres, comme Esperanza Aguirre, qui veulent faire un usage idéologique de l'humanisme ou d'une conception personnelle de l'homme.

Avant de s'intéresser au vrai débat sur l'utilisation de l'évaluation éducative, voici une synthèse des résultats de l'étude :

- $30 \%$ des élèves de 14 ans atteignent un niveau satisfaisant,

$-22,5 \%$ des élèves de 16 ans ont un niveau acceptable,

- la grande majorité des 14-16 ans a un niveau moyen, ni vraiment satisfaisant, ni vraiment insatisfaisant,

- très peu d'élèves sont très bons,

16 1. Rapport général (Résumé) ; 2. Résultats scolaires ; 3. Plans d'études et méthodes d'enseignement ; 4. Fonctionnement des centres ; 5 . Fonction professorale ; 6 . Famille et école. 
- la différence entre les élèves des réformes de 1970 et de 1990 n'est pas très significative,

- la corrélation entre niveau socioculturel et résultats scolaires reste évidente,

- les résultats sont légèrement supérieurs dans les écoles privées par rapport aux écoles publiques,

- le corps professoral se montre réticent face à l'évaluation et à la promotion automatique des élèves.

\section{Les intentions prévalent sur les résultats des évaluations}

Lors d'un entretien sur le diagnostic de l'éducation secondaire avec le directeur de l'INCE, un journaliste a posé directement les questions suivantes ${ }^{17}$ :

- Question : Est-il vrai que la ministre Aguirre aurait commandité cette évaluation afin de discréditer le système?

- Réponse : Non, je suis catégorique.

- Q. : Dans ce cas, y a-t-il eu des pressions pour altérer les résultats ?

- R. : S'il y en avait eu, ce qui est plus qu'improbable, la dynamique du travail scientifique les aurait éradiquées. Il y a eu, en revanche, une forte pression d'ordre psychologique afin que les résultats comparatifs ne soient pas révélés.

- Q. : Vous faites référence au droit de veto des communautés autonomes au sein de l'INCE ?

- R. : Oui.

- Q. : Certaines personnes dans l'entourage de Esperanza Aguirre pensent que la publication du Rapport dans notre journal est le fait des socialistes, qu'en pensez-vous?

- R. : Il est normal qu'un parti qui a réellement cru dans une loi la défende et tente d'apporter de l'eau à son moulin. Je ferais la même chose. J'ignore jusqu'à quel point le Parti socialiste ouvrier espagnol (PSOE) a été impliqué dans ces révélations. De toute façon, il serait ridicule de dire que l'enseignement secondaire obligatoire (ESO) se porte merveilleusement bien ou affreusement mal, parce que nous n'avons pas jugé l'ESO. En fait, on peut dire que la communauté éducative a plutôt une opinion positive à l'égard de l'ESO mais on ne peut pas mesurer avec exactitude les effets de l'ESO sur l'éducation espagnole. Ce serait prématuré.

Dans El País du 4 mars 1998, quotidien qui soutient officieusement la réforme et les gouvernements socialistes précédents, on peut lire les titres suivants : "le Rapport soutient la réforme, disent les parents, les syndicats et le PSOE. " La position plus impartiale du directeur contrebalance l'euphorie des parents et les critiques des détracteurs du gouvernement conservateur qui a inspiré

17 El País, 17 mars 1998, Éducation. 
l'étude : "Le directeur de l'étude (José Luís García Garrido) pense que les résultats ne sont pas dramatiques.»

Cette guerre des partis, des régions et des idéologues - par voie médiatique - se reflète dans les titres et les manchettes des journaux de l'époque :

- El Periódico (Madrid, 4 mars 1998) : «L'ESO réussit son premier examen : le rendement est identique voire supérieur dans plusieurs domaines comparativement à l'ancien système. L'INCE a examiné le niveau de 55000 élèves. [...] Les syndicats et les parents se félicitent du niveau supérieur de l'école publique.»

- El Faro de Vigo (Vigo, Galice, 4 mars 1998) : "Les connaissances des élèves de l'ESO de Galice sont supérieures à la moyenne nationale.»

- Hoy (Badajoz, Estrémadure, 4 mars 1998) : «Le rendement des élèves de l'ESO d'Estrémadure est inférieur à la moyenne nationale.»

- Ideal (Grenade, Andalousie, 4 mars 1998) : «Le rendement scolaire de l'ESO évite de justesse l'échec, selon la première étude officielle sur le sujet."

- La Vanguardia (Barcelone, Catalogne, 4 mars 1998) : "La première évaluation de l'ESO fournit de meilleurs résultats que le système précédent. "

- Ya (4 mars 1998) : "Le Rapport positif de l'ESO ridiculise la ministre Aguirre.»

- ABC (5 mars 1998) : "Ce n'est pas un désastre national, dit le Président du Conseil scolaire au sujet de l'ESO.»

- El País (5 mars 1998) : "60\% des professeurs estiment ne pas avoir reçu de bonne formation pédagogique."

- La voz de Asturias (Oviedo, 5 mars 1998) : «75\% des élèves ne savent pas que la Reconquête est partie des Asturies.»

- Ya (5 mars 1998) : "La réforme des humanités commence avec le consensus. La ministre Esperanza Aguirre a refusé de se prononcer sur le Rapport positif de l'ESO.»

- El Periódico (Barcelone, 6 mars 1998) : «La Catalogne a déjà obtenu de faibles résultats dans une étude sur le primaire.»

- ABC (7 mars 1998) : "Le Rapport sur le secondaire n'analyse pas que l'ESO mais également l'étape éducative de 14 à 16 ans. »

- El Mundo (7 mars 1998) : «M. Nasarre, secrétaire général de l'éducation et de la formation professionnelle, minimise l'importance du Rapport sur l'ESO qui, selon lui, analyse les connaissances des enfants de l'ancien système du secondaire.»

- El Periódico (7 mars 1998) : «Le Parti populaire tente de profiter de l'étude pour réformer la LOGSE. »

- La Vanguardia (7 mars 1998) : «M. Nasarre estime que le Rapport de l'ESO n'est qu'un diagnostic du système précédent. »

- ABC (8 mars 1998) : "Selon le responsable du Rapport de l'ESO, la qualité de l'enseignement espagnol est inquiétante. M. García Garrido, directeur de l'INCE, réclame davantage de moyens pour l'éducation.» résultat."

- El Periódico (Barcelone, 8 mars 1998) : «Jordi Pujol prévoit un bon 
- La Vanguardia (Barcelone, 8 mars 1998) : "La Catalogne revoit ses notes. L'échec à l'examen de l'ESO relance le débat sur la pédagogie catalane."

- $A B C$, (10 mars 1998) : "Le ministère souligne que le Rapport sur le secondaire est préoccupant mais pas alarmant."

- El País (10 mars 1998) : "Le ministère de l’Éducation et de la Science a chargé Arthur Andersen de faire un autre diagnostic. »

\section{Virulence de commentaires chez les politiciens, les professionnels et les professeurs}

Finalement, l'évaluation du secondaire montre que, paradoxalement, la zone méditerranéenne, moteur économique actuel, se situe au-dessous de la moyenne à côté de l'Estrémadure. Au contraire, les communautés autonomes de Madrid, Castille et León, Galice, Aragon et Rioja obtiennent les meilleurs résultats. Étant donné les caractéristiques de l'étude, le cadre géographique et l'organisation politique actuelle de l'État espagnol, le Rapport révèle des différences de connaissances en fonction des communautés. Les résultats redessinent une carte éducative qui divise, une fois de plus, le pays en deux. Sans simplifier, et sans donner plus de connotations à la séparation Nord/Sud, le secrétaire d'État a luimême ainsi répondu à la question des deux Espagne : "Étudier dans le Nord ou dans le Sud, ce n'est pas la même chose.»

Si l'on prend les résultats par région, l'Aragon, les Asturies, la Castille et León, la Galice, la Rioja, la Navarre, Madrid et le Pays Basque se situent dans le peloton de tête. Le wagon de queue comprend la Catalogne, les Baléares, Ceuta et Melilla, l'Estrémadure, la Murcie et Valence. Le groupe intermédiaire se compose de la Cantabria et de la Castilla-Mancha. À l'exception de l'Andalousie et des Canaries, situées géographiquement au Sud, qui ont refusé l'évaluation, une diagonale Nord/Sud, qui divise la péninsule ibérique en deux, peut être tracée du golfe de Lyon (France) au cap de Saint Vincent (Portugal). Il y a plus d'un siècle, cette même carte divisait mutatis mutandis la scolarité du pays en deux : le Nord plus alphabétisé et le Sud avec un pourcentage d'analphabétisme plus élevé. Ce n'est ni le moment de faire des analyses socio-économiques, ni de commenter les résultats d'une évaluation du système éducatif secondaire aux facteurs multiples. À chacun son interprétation.

En conclusion, après tant de polémiques utilisées à des fins de luttes politiques entre les gouvernements régionaux et le gouvernement d'État, en fonction des affiliations et des partis respectivement au pouvoir, nous ne croyons pas que la qualité de l'éducation du système ait pu être mesurée à travers les 
chiffres inscrits dans ce macro-rapport. Il est évident qu'après tant d'expectatives suscitées dans le contexte éducatif espagnol, aucun des groupes intéressés, à commencer par le gouvernement, n'a pu être satisfait par ce Rapport. Nous pouvons cependant apprécier le bon travail technique des évaluateurs.

En définitive, lorsqu'il s'agit d'évaluer l'homme une question se pose : qui peut s'arroger la faculté de l'évaluer ? L'évaluation du système éducatif en pose une autre : qui détermine les critères de qualité ? Si la qualité consiste en l'efficacité, la réponse est clairement exprimée dans la performativity de Lyotard : "Soyez opérationnels ou disparaissez »... comme les «inopérants» de l'époque Van Gogh ou Cervantès, dont l'effectivité ou la "qualité » n'a pas été reconnue tout de suite. C'est ça, le temps de l'évaluation? 